\title{
The structure of a penumbral connection between solar pores
}

\author{
J. Hirzberger ${ }^{1,4}$, S. Stangl ${ }^{1}$, K. Gersin ${ }^{1}$, J. Jurčák ${ }^{2}$, K. G. Puschmann ${ }^{3}$, and M. Sobotka ${ }^{2}$ \\ ${ }^{1}$ Institut für Physik, IGAM, Universitätsplatz 5, 8010 Graz, Austria \\ 2 Astronomical Institute, Academy of Sciences of the Czech Republic, 25165 Ondřejov, Czech Republic \\ 3 Universitäts-Sternwarte, Geismarlandstraße 11, 37083 Göttingen, Germany \\ 4 Max-Planck-Institut für Sonnensystemforschung, 37191 Katlenburg-Lindau, Germany \\ e-mail: hirzberger@mps.mpg.de
}

Received 18 April 2005 / Accepted 17 June 2005

\begin{abstract}
High resolution 2D-spectro-polarimetric observations have been used to analyse the magnetic field and flow topologies of a penumbral connection between two opposite polarity solar pores. A filamentary structured Evershed-like material flow from one pore to the other along the magnetic field lines has been detected. The flow channels are co-spatial with bright penumbral filaments close to the pore which feeds the flow and the clear brightness-velocity relation vanishes close to the pore which represents the sink of the flow. The boundary between umbra and penumbra of the two pores show significant differences: bright comet-like penumbral grains represent endings of penumbral filaments at the flow sources whereas no such grains were found at the sinks of the flow. Furthermore, a systematic variation of the asymmetries of measured Stokes $V$ profiles across the penumbral connection have been found. The obtained results are in accordance with the widely-accepted uncombed penumbra hypothesis and the moving flux tube model.
\end{abstract}

Key words. Sun: photosphere - Sun: magnetic fields - Sun: sunspots

\section{Introduction}

Sunspots reveal a variety of visual structures and magnetic field topologies, ranging from unipolar singular pores to complex spot groups including opposite polarities even within the same penumbral surrounding ( $\delta$-spots). Opposite polarities are also often observable in adjacent pores. Frequently, pores are surrounded by a more or less rudimentary penumbra. The formation processes and the dynamics of penumbral structures are still a matter of debate and many details remain unclear or contradictory. (A recent overview about the knowledge of sunspots and a detailed list of references has been given by Solanki 2003).

Penumbrae are characterised by a pattern of bright and dark fibrils which are usually oriented parallel to the radius vector of the spots. Sütterlin (2001) pointed out that penumbral fibrils have a preferred width of about $250 \mathrm{~km}$. Recent observations (Scharmer et al. 2002; Rouppe van der Voort et al. 2004), however, suggest that the width of these fibrils is $100 \mathrm{~km}$ or even less. The magnetic field topology in sunspot penumbrae is believed to exhibit an "uncombed" structure (see e.g. Degenhardt \& Wiehr 1991; Schmidt et al. 1992; Solanki \& Montavon 1993; Martínez Pillet 2000; Bellot Rubio et al. 2004), i.e. almost horizontal magnetic flux tubes are embedded in an environment of inclined field lines. An alternative model has been presented by Sánchez Almeida (1998): based on the measured broad band circular polarisation, he provides evidence that the magnetic fields may fluctuate on scales between 1 and $15 \mathrm{~km}$.

An important dynamical phenomenon appearing in sunspot penumbrae is the Evershed flow, i.e. an outward directed motion detectable by Doppler shifts in out-of-disk-centre sunspots. According to Title et al. (1993) and Bellot Rubio et al. (2004) the bulk of the Evershed motion is located in the horizontal flux tube component of the penumbra. Less accordance has been achieved so far in the relation of the magnetic field and flow topologies with the penumbral intensity pattern. Several authors (Schmidt et al. 1992; Wiehr 2000; Westendorp Plaza 2001) have found that dark filaments correspond to the more horizontal field component and it has been stated (e.g. Schröter 1965; Stellmacher \& Wiehr 1971; Title et al. 1993; Shine et al. 1994; Rimmele 1995a; among others) that the Evershed flow is associated with these dark penumbral filaments. Beckers \& Schröter (1969), Abdussamatov (1976) and Wiehr (2000) have measured an increased field strength in the dark filaments. However, other studies have pointed out deviations from this picture: e.g. Wiehr \& Stellmacher (1989), Lites et al. (1990), and Hirzberger \& Kneer (2001) could not confirm a clear correlation between the flow velocity and the intensity pattern. Hofmann et al. (1994) found no correlation of the fluctuations of the field strength with the intensity fluctuations and Westendorp Plaza et al. (2001) have estimated higher field strengths in regions of hotter gas. 
From a theoretical point of view the Evershed flow might be explained by a siphon model (Meyer \& Schmidt 1968; Thomas 1988; Montesinos \& Thomas 1993, 1997). A numerical simulation of flow channels has been presented by Schlichenmaier et al. (1998). In this model the flow is concentrated within thin flux tubes which rise vertically from the solar interior in the inner penumbra and bend to become almost horizontal in the visible photosphere. At the position where the flux tubes bend, bright inward-moving penumbral grains should be visible. The inward motion of these penumbral grains, located in the inner penumbra, is in good agreement with observational results (Muller 1973; Tönjes \& Wöhl 1982; Sobotka et al. 1999; Sobotka \& Sütterlin 2001). Vertical upflows at the inner penumbra have been measured by e.g. Johannesson (1993), Rimmele (1995a,b) and Hirzberger \& Kneer (2001).

The moving flux tube model of Schlichenmaier et al. (1998) is in good agreement with the uncombed penumbra model of Solanki \& Montavon (1993) if the flux tubes are considered to be embedded in a homogeneous more vertically orientated background field. This agreement has been demonstrated by observations (Schlichenmaier \& Collados 2002) as well as by inversions (Borrero et al. 2004) and model simulations (Müller et al. 2002) of Stokes profiles. Particularly, Stokes $V$ profiles show a systematic variation of their shapes which can be adequately explained by two- or more-component uncombed penumbra models. Usually the appearance of asymmetrical Stokes profiles has been explained by the so-called crossover effect (see e.g. Grigorjev \& Katz 1972; Sánchez Almeida \& Lites 1992). This effect is based on a simultaneous occurrence of gradients in field inclination and flow velocity, e.g. due to insufficient spatial resolution.

This paper analyses the structure of a penumbral connection between two solar pores by means of two-dimensional (2D) spectro-polarimetric observations. Common properties as well as different features of this structure, compared to "ordinary" sunspot penumbrae, will be outlined. After a description of the observations and data reduction procedures (Sect. 2) the magnetic field and flow structure of the miscellaneous white light phenomena will be discussed (Sect. 3). In Sect. 4 conclusions in the scope of various model assumptions will be drawn.

\section{Observations and data reduction}

The data were obtained on June 21, 2004 with the "Göttingen" Fabry-Pérot Interferometer (Bendlin et al. 1992; Koschinsky et al. 2001) which is installed at the German Vacuum Tower Telescope on Tenerife, Canary Islands. A Stokes $V$ polarimeter (see Volkmer 1995) consisting of a $\lambda / 4$ retarder plate and two crossed calcites was installed in front of the narrow band camera. This device effects a spatial separation of left and right circularly polarised beams $(I+V) / 2$ and $(I-V) / 2$ on the CCD array. We have obtained a time series of narrow band scans across the Fe I $6301.5 \AA$ and the Fe I $6302.5 \AA$ lines. Each scan consists of 50 wavelength positions ( 24 positions at the Fe I $6301.5 \AA$ line and 26 positions around the Fe I $6302.5 \AA$ line, including a terrestrial oxygen line blend) separated by $\Delta \lambda=26.47 \mathrm{~m} \AA$ and 3 images at each position have been stored.
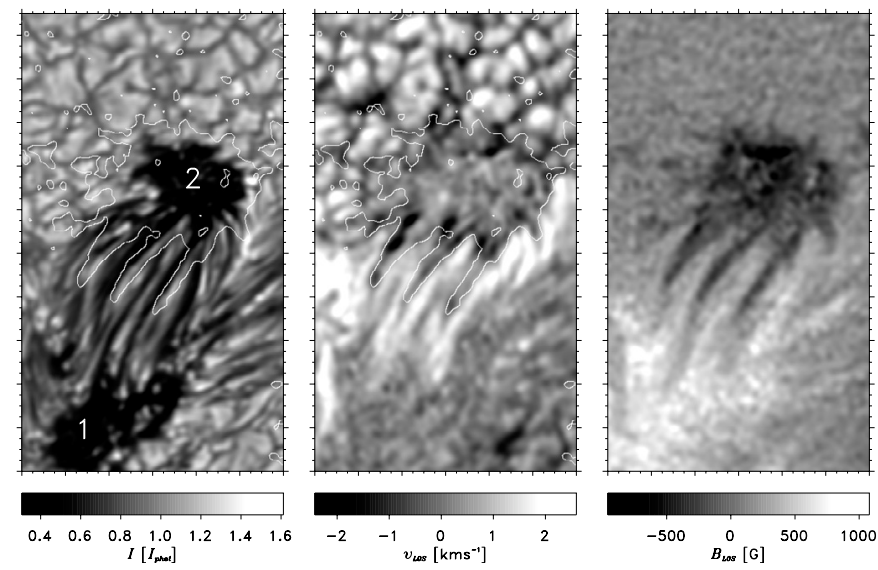

Fig. 1. Speckle reconstructed broad band image (left panel) of the observed solar region. The middle and right panels exhibit line-of-sight Doppler velocities, $v_{\text {LOS }}$ (positive values denote blueshifts) and magnetic field strengths, $B_{\mathrm{LOS}}$, obtained from the centres of gravity of the Fe I $6301.5 \AA$ line. White contours mark the magnetic neutral lines. Tickmarks are at 0.5 distance.

The filter width was set to $44 \mathrm{~m} \AA$ ( $F W H M$ ) and the time separation between subsequent is $51.6 \mathrm{~s}$.

Simultaneously with the narrow band scans, bursts of broad band filtergrams around $6302 \AA \pm 50 \AA$ ) were obtained. All the data were carefully corrected for dark currents and flat fields and the narrow band scans were additionally corrected for the transmission curve of a narrow band interference filter which was installed in front of the Fabry-Pérots. Broad band data were reconstructed for the influences of the Earth's atmosphere using speckle interferometric techniques (de Boer 1993) based on the speckle masking and spectral ratio methods (von der Lühe 1984; Weigelt 1977). Narrow band data were reconstructed by using the optical transfer functions of the atmosphere as obtained from the simultaneously-recorded broad band data (see Krieg et al. 1999).

The time series of reconstructed broad band images was carefully aligned and destretched by using a correlation tracking code as presented in Yi \& Molowny Horas (1992). The obtained global shifts and distortions were also used to align the narrow band data. The final step was the application of a subsonic filter (see Title et al. 1989) with a cut-off phase velocity of $5 \mathrm{~km} \mathrm{~s}^{-1}$ to both broad band as well as narrow band data in order to remove contributions of five-minute-oscillations. We obtained a $24 \mathrm{~min}$ long time series of 28 broad band images and 2D-spectra with sizes of $120 \times 210$ pixels. The size of one pixel corresponds to 0 !' 1 on the solar disk.

Figure 1 shows one of the final broad band images (intensities in units of the mean photospheric intensity, $I_{\text {phot }}$ ) together with the simultaneous Dopplergram (line-of-sight flow velocities, $v_{\mathrm{LOS}}$ ) and the magnetic field map (line-of-sight field strengths, $B_{\mathrm{LOS}}$ ) as obtained from the Fe I $6301.5 \AA$ line. The Doppler shifts and field strengths were obtained by computing the centres of gravity (see e.g. Semel 1967) of the measured Stokes $I+V$ and $I-V$ profiles. Stokes $V$ profiles were extracted by a subtraction of the $I+V$ and $I-V$ components (see Sect. 3.4). In order to improve the polarimetric accuracy a second order flat fielding procedure according to 


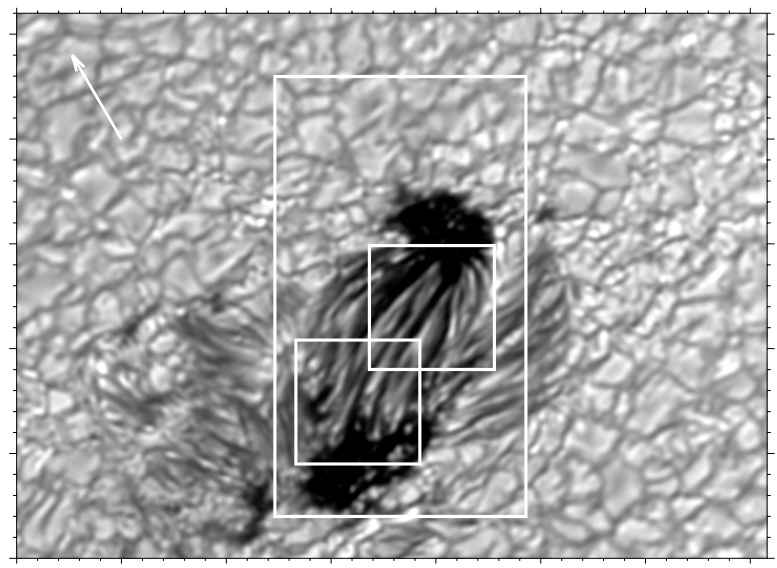

Fig. 2. Original-size speckle reconstructed broad band image showing the entire penumbral region around the two pores of our FOV. The large box borders the subfields shown in Fig. 1 (narrow band data), the upper small box is cospatial with the righthand panels of Fig. 3, and the lower box defines the lefthand panels of Fig. 3 and the cutouts in Fig. 5. The white arrow points towards disk centre. Tickmarks are at $1^{\prime \prime}$ distance.

Sánchez Almeida \& Martínez Pillet (1994) was applied. A detailed description of the calculation of these parameters is given in Stangl \& Hirzberger (2005). The zero reference point of the Doppler maps was defined by averaging the Doppler shifts in the darkest regions $\left(I<0.5 I_{\text {phot }}\right)$ of the lower pore in our field of view (FOV). Since we do not expect an angular resolution better than 0.3 the Doppler and magnetic field maps were smoothed by $3 \times 3$ pixels. The (rms) noise levels in the resulting $v_{\mathrm{LOS}}$ and $B_{\mathrm{LOS}}$ maps have been estimated from the corresponding power spectra and are approximately $50 \mathrm{~m} \mathrm{~s}^{-1}$ and $70 \mathrm{G}$, respectively. However, instrumental polarisation effects ( $Q, U \rightarrow V$ crosstalk) may considerably influence the magnetic field measurements. From comparable observations performed by Bello Gonzalez (2004) these effects were estimated as approximately $20 \%$.

In our FOV two pores connected by a penumbral structure are visible. The pores are located just beside a mature sunspot (NOAA 0634) at a heliocentric angle of $\cos \theta=0.87$. The direction towards this sunspot is along the negative $y$-axis in Fig. 1. The direction towards the disk centre is marked by the white arrow in Fig. 2.

\section{Results}

\subsection{General properties}

From SOHO/MDI magnetograms it can be found that the lower pore in Fig. 1 (hereafter "pore 1") has the same magnetic polarity as the neighbouring large spot whereas the upper one (hereafter "pore 2") shows the opposite magnetic polarity. From our data we obtain maximum field strengths (using the $\mathrm{Fe}$ I $6301.5 \AA$ line) of approximately $1240 \mathrm{G}$ in pore 1 and about $-1290 \mathrm{G}$ in pore 2 . These values are rather low, which might be due to the following reasons: (i) since we have obtained only three narrow band images per spectral position the noise in the reconstructed data is rather high, particularly in the dark umbrae where the intensity is low; (ii) the data are not corrected for stray light, which might reduce the measured magnetic field strengths; (iii) the penumbral structures are orientated almost perpendicularly to the solar radius vector; additionally the pores are almost $30^{\circ}$ out of disk centre. Therefore, the measured line-of-sight component of the field vector $B_{\mathrm{LOS}}=|B| \cos \theta_{\mathrm{LOS}}$, where $\theta_{\mathrm{LOS}}$ denotes the field inclination to the line of sight, might be significantly lower than the actual field strength $|B|$; (iv) instrumental polarisation effects may lead to an underestimation of the field strengths.

The measured Doppler velocities are in good accordance with the granular intensity pattern in the quiet region above pore 2 (see Fig. 1). We use a sign convention so that positive velocities denote blueshifts and negative values correspond to redshifts. To the right-hand side of pore 1 the broad band image shows a disturbed granular pattern. In the Doppler map, however, indications for a cellular pattern are barely visible in this region. This holds for the entire $24 \mathrm{~min}$ time series. Since this region is in between the pores and the nearby large spot both the flows and the intensity pattern might be modified by magnetic fields. Positive field strengths in this region are clearly visible in the magnetic field maps whereas in the quiet granular region above pore 2 the field strengths do not exceed the noise level of our data (cf. Fig. 1). A significant modification of the flow structure has been reported by Hirzberger (2003) for a region close to a rudimentary penumbra of a large pore.

The penumbral connection between the two pores in the FOV shows a pronounced filamentary structure also in the flow maps. In the lower part of the penumbral connection the Doppler maps are characterised by mainly upward velocities with a tendency to increase with increasing brightness of the penumbral filaments. In the upper part of the structure both upflows and downflows are visible. The separation between upward and downward motions is well defined by the magnetic neutral line. The line-of-sight velocities in the penumbral connection are approximately within the intervals $-3.1 \mathrm{~km} \mathrm{~s}^{-1}<v_{\mathrm{LOS}}<2.9 \mathrm{~km} \mathrm{~s}^{-1}$ for the Fe I $6301.5 \AA$ line and $-4.4 \mathrm{~km} \mathrm{~s}^{-1}<v_{\mathrm{LOS}}<3.4 \mathrm{~km} \mathrm{~s}^{-1}$ for the Fe I $6302.5 \AA$ line. The above outlined findings might be interpreted as an Evershed-like material flow parallel to the magnetic field lines from pore 1 to pore 2 .

Further penumbral structures appear in our FOV on both sides of the penumbral connection between the pores (see Fig. 2). These penumbrae are partially rooted in the lower pore. The outer boundaries of these structures are located outside of the FOV of the narrow band images. In the original-size broad band images several micropores embedded in granular regions can be seen there. In the small part visible in the narrow band images the penumbral filaments are almost perpendicularly orientated to the solar radius vector, so that the line-of-sight flow velocities are rather low there.

\subsection{Fine structure of the flow geometry}

Usually the term "Evershed flow" is assigned to a flow field which is directed more or less horizontally outward in a sunspot penumbra. Close to the umbral boundary of pore 2 this flow 

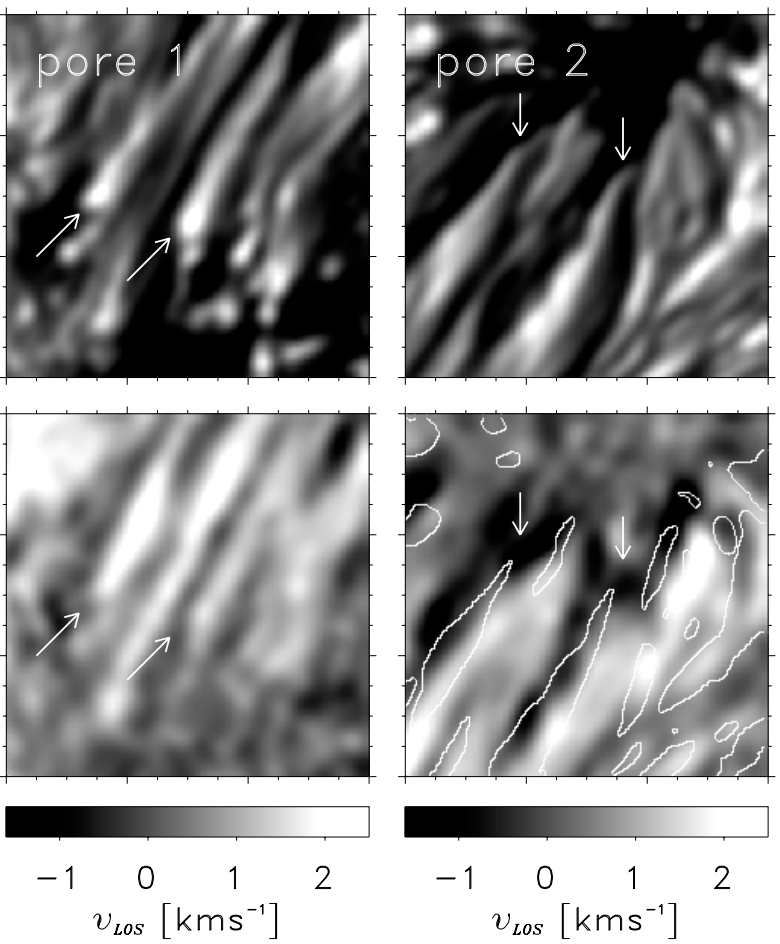

Fig. 3. Enlarged subfields of one the broad band images (upper panels) and corresponding Doppler maps (lower panels). Left panels: endings of the penumbral filaments (peripatopause) of pore 1; right panels: peripatopause of pore 2 . The white contours in the lower right panel denote broad band intensities of $0.9 I_{\text {phot }}$. Tickmarks are at 0.5 distance.

points in the opposite direction. Therefore, the boundary between penumbra and umbra (peripatopause) of this pore might show some differences to a common peripatopause, as e.g. the one in pore 1. An obvious difference between the peripatopauses of the two pores is that the endings of the bright penumbral filaments at pore 1 in most instances exhibit bright grains giving the filaments a comet-like (cf. Muller 1973) or tongue-like (cf. Grossmann-Doerth et al. 1986) appearance. In pore 2 no such grains are visible. Here the endings of the bright penumbral filaments are mostly defined by a monotonic reduction of their widths. This difference is clearly visible in enlarged sub-boxes of the broad band images as shown in Fig. 3 . Note that the two bright structures visible in the broad band image of pore 2 are bright umbral dots (upper right panel of Fig. 3, positions $\left[0.3,5^{\prime \prime} 7\right]$ and $\left.\left[0.4,4{ }^{\prime \prime} 2\right]\right)$. At least one of them corresponds to a fragment of a bright point penetrating into the umbra from the nearby granular field.

The corresponding flow maps also show significant differences (Fig. 3, lower panels). Close to pore 1 the bright filaments correspond to blueshifts (upflows). The two overplotted arrows point to the same positions in both the broad band and the Doppler image. However, the maximum velocity (up to $2.33 \mathrm{~km} \mathrm{~s}^{-1}$ in the filament marked with the left arrow) does not appear at the brightness maximum of the penumbral grain but approximately $2^{\prime \prime}$ away from it. This result is in excellent agreement with the numerical simulations of Schlichenmaier et al. (1998). In this model the Evershed flow is assumed to
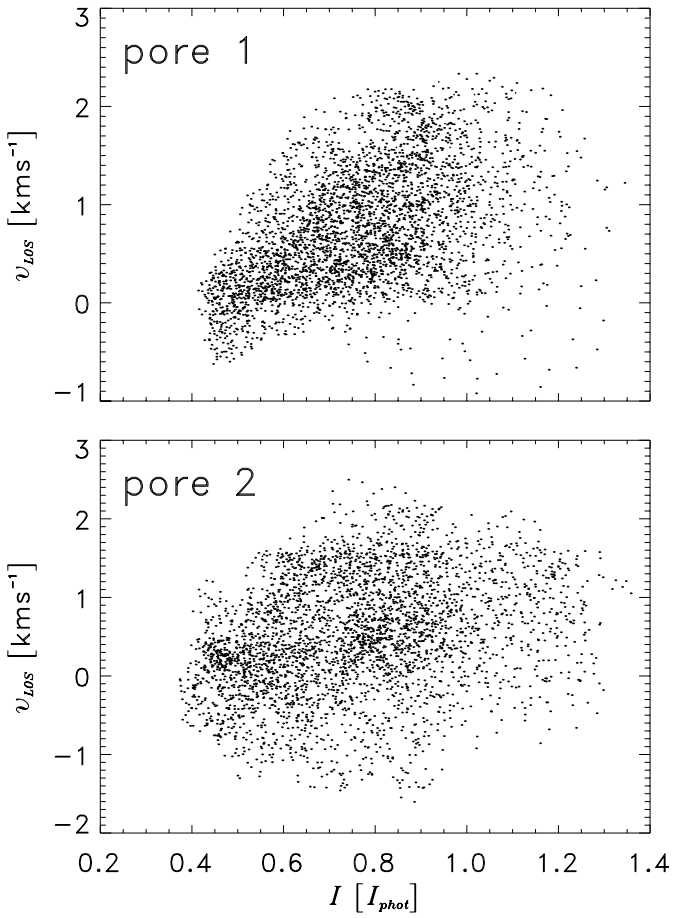

Fig. 4. Scatter plots of the subfields shown in Fig. 3.

arise in bright grains where vertical flux tubes are bent to the horizontal. At the footpoints of the horizontal flux tubes (i.e. at position of the bending) the temperature is found to exceed the mean penumbral temperature by several $10^{3} \mathrm{~K}$. The formation of penumbral grains might then be related to this temperature excess. The flow velocities in the horizontal flux tube are expected to increase monotonically from the footpoint towards larger distances. This is also clearly visible in our flow maps. The absolute velocity gradient must be considered to be much steeper than that expected from Fig. 3 since only the line-ofsight components of the flow velocities can be estimated from our data.

At pore 2 the endpoints of the bright penumbral filaments (again marked by white arrows) are situated in regions of localised strong redshifts. However, neither the bright nor the dark filaments are correlated with the flows in this entire subfield. Examining the areas within the overplotted white contours in the Doppler map shows that all the bright penumbral filaments are located in regions where the flow velocities change from positive to negative values. This lack of correlation is substantiated in the scatter plots of Fig. 4: whereas in the upper panel (pore 1) a positive correlation (correlation coefficient $c=0.47$ ) between broad band intensity and Doppler velocity might be guessed, in the lower panel (pore 2) the scatter plot looks more uncorrelated $(c=0.29)$.

\subsection{Time evolution of penumbral filaments}

According to the numerical model of Schlichenmaier et al. (1998) an inward migration of the bright footpoints of the Evershed flow should be visible. The temporal evolution of the peripatopause of pore 1 is displayed in Fig. 5. For almost all 


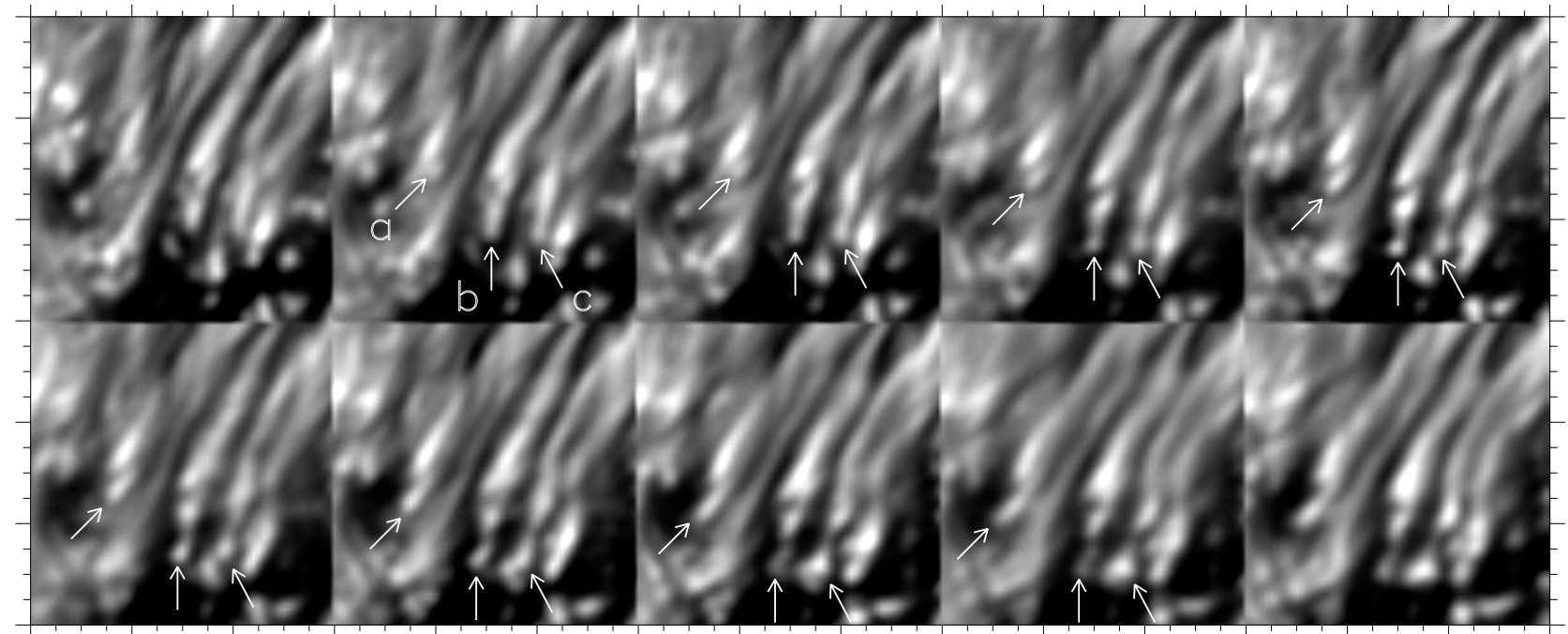

Fig. 5. Temporal evolution of the penumbral filaments connected to pore 1 (from left to right and top to bottom). The arrows mark three inward moving penumbral grains. Tickmarks are at 0 '. 5 distance; the time interval between subsequent images is $103 \mathrm{~s}$.

of the bright penumbral grains in the subfield this inward migration can be seen. Particularly the three examples marked by white arrows can be followed easily and confirm the results of Schlichenmaier et al. (1998).

An additional effect visible in Fig. 5 is a separation of some of the bright grains from their original penumbral filaments as they enter into the umbra. Whereas the penumbral grain marked as example "a" in Fig. 5 moves without further separation, this effect is clearly observable for examples " $\mathrm{b}$ " and "c". The resulting structures after separation from the penumbral filaments resemble peripheral umbral dots (for details about nomenclature and physical properties see e.g. Grossman-Doerth et al. 1986; Sobotka et al. 1997). They can even merge with each other (example "c"). Against this background it might be expected that the umbral-dot like structures originating from penumbral grains are related to upwardmoving gas flows as found for penumbral grains. This conclusion would additionally support theoretical and numerical models of e.g. Parker (1979), Choudhuri (1986), Knölker \& Schüssler (1988), and Degenhardt \& Lites (1993). Therefore, we have measured the Doppler velocities and magnetic field strengths of all bright penumbral grains and umbral dots visible in and around pore 1. Figure 6 shows these quantities averaged in $3 \times 3$ pixel boxes centred on the brightest pixel of each structure vs. broad band intensity. The umbral dots can be discriminated from the penumbral grains by lower broad band intensities. The magnetic field strength shows a decreasing linear trend from the darkest umbral dots towards the brightest penumbral grains, thus indicating a smooth transition between both phenomena. The field strengths of the bulk of the structures are below the average umbral field strength.

The Doppler velocities, however, show rather different behaviours for the two types of structures. For most of the detected umbral dots the measured velocities amount to small positive values (upflows). A slightly increasing trend towards brighter structures can be inferred. The zero reference value has been defined by the average velocity of the darkest regions
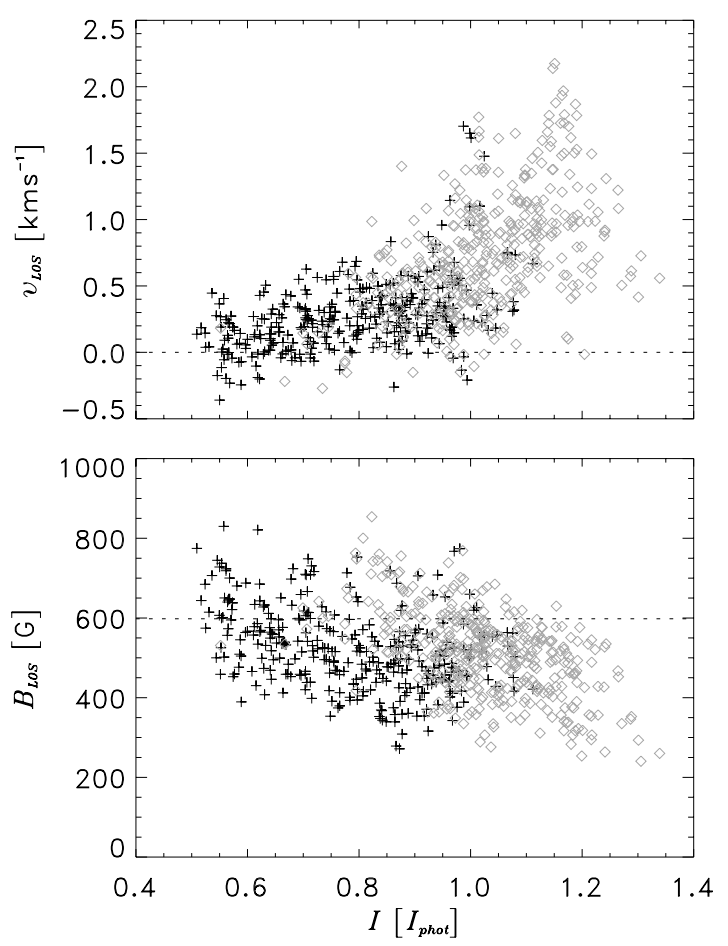

Fig. 6. Scatter plots of $v_{\mathrm{LOS}}$ and $B_{\mathrm{LOS}}$ vs. broad band intensity, $I$, for penumbral grains (diamonds) and umbral dots (plus signs) in and around pore 1. Dotted lines denote mean values of $v_{\mathrm{LOS}}$ and $B_{\mathrm{LOS}}$ in the darkest regions with $I<0.5 I_{\text {phot }}$.

in pore 1 . Thus, the orientation of the flows - with respect to the dark umbra - is mainly upward. The velocity distribution of the penumbral grains is, in contrast to that of the umbral dots, less uniform, i.e. the scatter is much larger. Nevertheless, almost all of these structures show strong upflows and also here a tendency for an augmentation of the Doppler velocities towards brighter grains. This trend is, however, much steeper than that for the umbral dots. The maximum value of $2.18 \mathrm{~km} \mathrm{~s}^{-1}$ belongs to a penumbral grain with $I=1.15 I_{\text {phot }}$. This grain is 

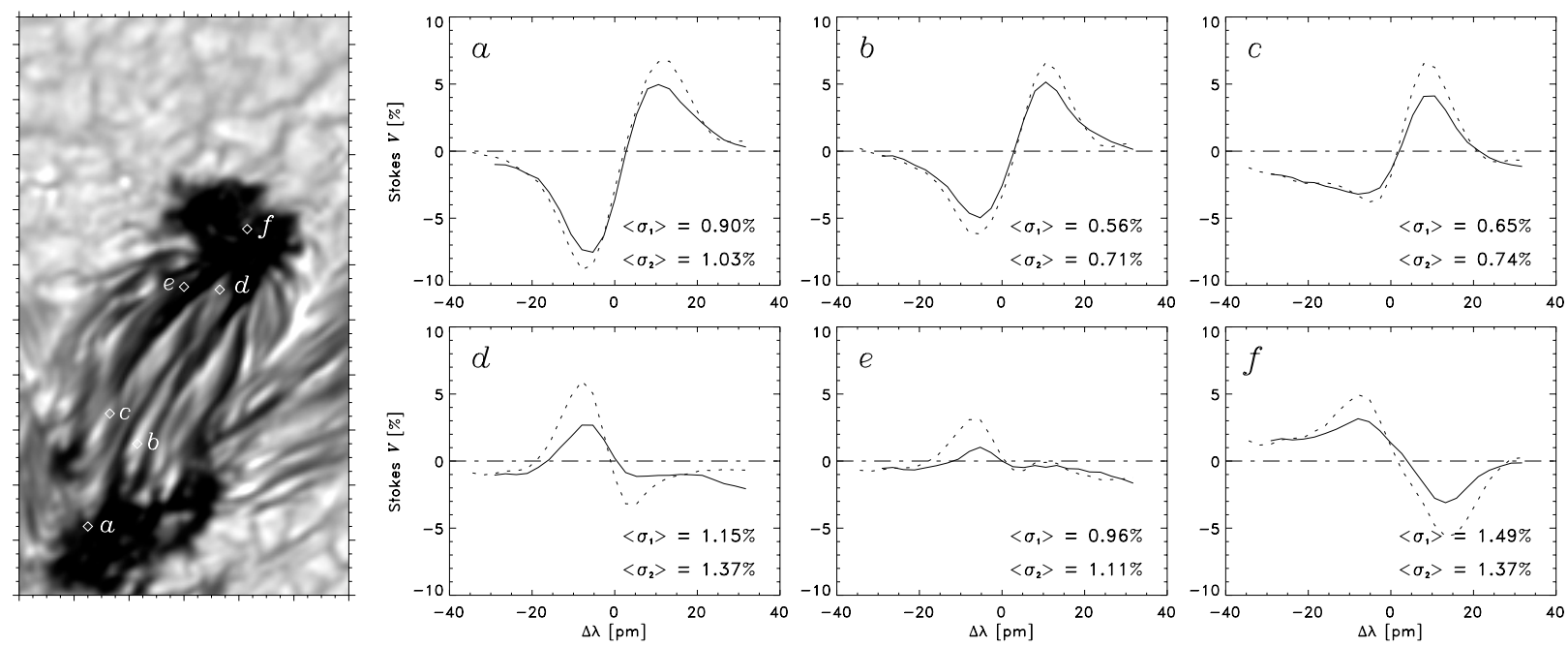

Fig. 7. Time-averaged Stokes $V$ profiles of 6 selected positions in the FOV. Solid lines correspond to the Fe I $6301.5 \AA$ line, dotted lines are computed from the Fe I $6302.5 \AA$ line, respectively. Panel labels correspond to the marked positions in the time averaged broad band image (left panel). The quantities $\left\langle\sigma_{1}\right\rangle$ and $\left\langle\sigma_{2}\right\rangle$ denote the spectral averaged standard deviations of the mean $V$ profiles.

considerably fainter than the brightest ones with intensities up to $1.34 I_{\text {phot }}$.

\subsection{Stokes $V$ profiles}

A visualisation of the structure of the penumbral connection can be obtained from the variation of the shapes of the Stokes $V$ profiles across the FOV. However, since individual profiles may have low significance in Fig. 7, at first, we show time averaged profiles of selected positions. Temporal means were obtained by averaging the $V$ profiles of each pixel throughout the time series. The penumbral structure is rather stable in time, as can be seen from the time-averaged broad band image (Fig. 7, left panel). Correspondingly, $V$ profiles from individual spectral scans show, aside from higher noise levels, similar shapes to those plotted in Fig. 7. This fact is also reflected by the low standard deviations of the averaged profiles. The quoted values $\left(\left\langle\sigma_{1}\right\rangle\right.$ for the Fe I $6301.5 \AA$ line, $\left\langle\sigma_{2}\right\rangle$ for the Fe I $6302.5 \AA$ line) represent spectral averages of the temporal variations of the corresponding $V$ profiles.

The basic feature of the $V$ profiles plotted in Fig. 7 is that the amplitudes calculated from the Fe I 6302.5 $\AA$ line are higher everywhere than those estimated from the Fe I $6301.5 \AA$ line although their shapes are rather similar. This may be attributed to the smaller Landé factor and the somewhat greater formation height of the Fe I $6301.5 \AA$ line. In the umbrae (profiles "a" and "f") the $V$ profiles are more or less ordinary, i.e. antisymmetrically shaped although much more fluctuating in pore 2 than in pore 1. A few arcseconds away from the pores the profiles exhibit a significant asymmetry, i.e. one of the lobes becomes lower and broader than the other one. In the lower part of the umbral connection (profile "c") the blue lobes show this behaviour, in the upper part (profiles "d" and "e") the red lobe is weakened. In the region close to the magnetic neutral line (cf. Fig. 1) the Stokes $V$ amplitudes are rather low and profiles show mostly three lobes. The low amplitudes are expected since the line-of-sight components of the field should be small here. The appearance of three-lobed Stokes $V$ profiles close to the magnetic neutral line is in good agreement with Schlichenmaier \& Collados (2002). However, the transversal field components are expected to exceed the longitudinal (line-of-sight) component. Consequently, crosstalk from Stokes $Q$ and $U$ towards Stokes $V$ may constitute a significant contribution for the measured Stokes $V$ profiles. All features described above appear in both bright and dark filaments, however, sometimes at different distances from the umbrae. Generally, the $V$ profiles show a smooth variation of shapes along single filaments, whereas the variation transverse to the filaments is much more fluctuating, i.e. adjacent positions from bright and dark filaments often reveal rather diverse shapes.

Artificial penumbral Stokes $V$ profiles as modelled e.g. by Müller et al. (2002) show rather different shapes to the ones obtained from our data. The simulated profiles show many details of all included model components which will are hidden in the observations by noise and by the finite spectral resolution. Nevertheless, an almost complete disappearance of one of the lobes occurs also in the simulations if a line-of-sight inclination angle of $30^{\circ}-40^{\circ}$ is assumed (cf. Fig. 7 in Müller et al. 2002). The disappearance of one of the lobes is explained by the cancellation of the blue lobe of the background component by the red lobe of a Doppler-shifted flux tube component. For our observed profiles this means that at least two magnetic field components within the resolution elements are necessary to explain the results: Close to pore 1 the obvious strong blueshift in one of the components may cancel the blue lobe of the measured profile; close to pore 2 the measured redshifts may lead to a cancellation of the red lobe.

Figure 8 shows non-averaged Stokes $V$ profiles extracted from the bright filament marked by the left white arrows in the upper panels of Fig. 3. The $V$ profiles displayed in Fig. 8a stem from the bright grain at the ending of the filament and show the ordinary two-lobed antisymmetric shape (see also profiles "b" in Fig. 7). The amplitudes are rather high so that it may be concluded that a field component with a small inclination 

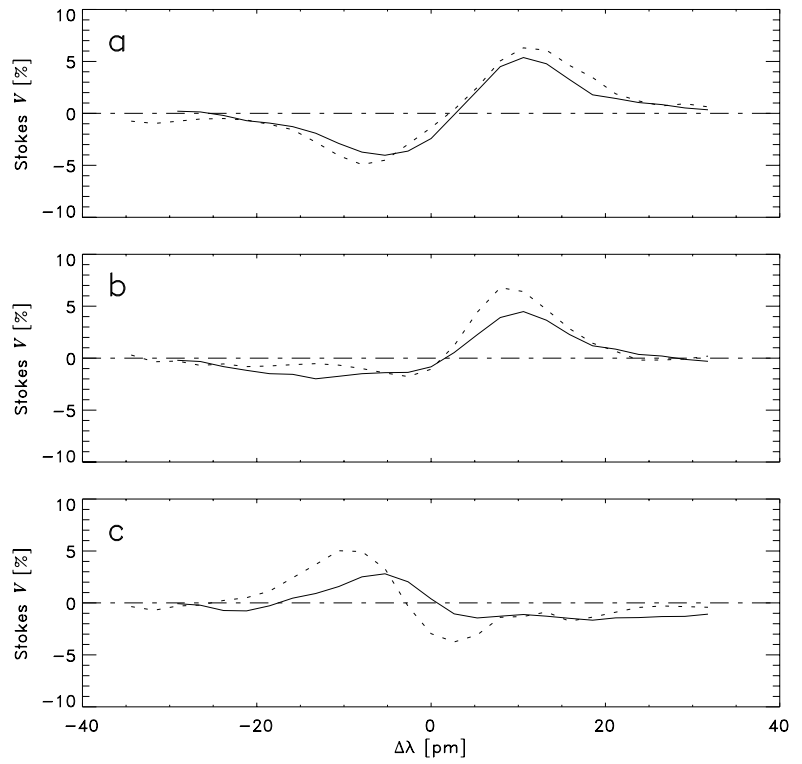

Fig. 8. Stokes $V$ profiles from three positions along a single bright penumbral filament (marked by the leftmost white arrows in the two upper panels of Fig. 3). Solid lines correspond to the Fe I $6301.5 \AA$ line, dotted lines stem from the Fe I $6302.5 \AA$ line, respectively.

angle with respect to the line of sight dominates the formation of the profiles in this resolution element. Figure $8 \mathrm{~b}$ shows the $V$ profiles in the filament approximately 1 '. 75 away from the bright grain. Here the weakening of the blue lobe is well pronounced for both spectral lines, whereas in Fig. 8c (another 3.' 19 along the bright filament, i.e. close to pore 2) the red lobes are reduced. Antisymmetrical profiles - as common in the bright penumbral grains of the peripatopause of pore 1 (e.g. Fig. 8a) - have not been found in the endings of the penumbral filaments close to pore 2. Even in the umbra of pore 2 a large fraction of the obtained profiles exhibit a considerable asymmetry. This results in a significantly higher standard deviation of the time-averaged profiles of the umbra of pore 2 as obtained in pore 1 (cf. profiles labelled with "a" and "f" in Fig. 7).

\section{Conclusions}

The present high resolution study of a penumbral connection between two pores shows a large variety of features, parts of them well known from "ordinary" sunspot penumbrae, others not observed there. From the magnetic field and Doppler maps it may be concluded that an Evershed-like flow takes place parallel to the field lines from pore 1 towards pore 2 i.e. crossing the magnetic neutral line. This result is unlike the situation in large $\delta$-sunspots as analysed by Lites et al. (2002). These authors found a converging zone where the flow direction of the Evershed motion reverses at the magnetic neutral line. Both the results of the present study as well as those of Lites et al. (2002) are in good agreement with the model assumption of an "uncombed" or "fluted" penumbral structure (cf. e.g. Solanki \& Montavon 1993). Nevertheless, alternative explanations might be adopted from micro-structured atmosphere models (e.g. Sánchez Almeida \& Bonet 1998).
The properties of the observed penumbral grains in the peripatopause of pore 1 (intensity excess, inward motion, upward directed gas flows and ordinary-shaped Stokes $V$ profiles) confirm the suggestion that they represent footpoints of flux tubes as simulated by Schlichenmaier et al. (1998). Since it is frequently observed that penumbral grains detach from the filaments, they might have similar properties to peripheral umbral dots. Analysing magnetic field strengths and broad band intensities, a smooth transition from penumbral grains to umbral dots is found. The Doppler velocities, however, show obvious differences in these two types of phenomena.

A fiercely debated topic concerns the correlation of brightness, field strengths and flow velocities of the penumbral filaments. In the majority of studies (Schröter 1965; Stellmacher \& Wiehr 1971; Title et al. 1993; Shine et al. 1994; Rimmele 1995a) a preference for the Evershed flow to be situated in the dark filaments has been assumed. Several authors (Wiehr \& Stellmacher 1989; Lites et al. 1990; Hirzberger \& Kneer 2001), however, have stated deviations from this picture. In the present study the Evershed flow is clearly related to bright filaments in the lower part of the penumbral connection, i.e. where the inclination angles, $\theta_{B}$, of the flow and field vectors are assumed to remain below $90^{\circ}$. In the upper part of the structure, i.e. where the flow and field vectors may reach inclination angles above $90^{\circ}$, no clear correlation between intensities and flow velocities has been found. The magnetic neutral line, however, clearly separates blueshifted from redshifted regions. Similar results have been obtained by Bello González et al. (2004). They found a tendency for the Evershed flows to be located in bright penumbral filaments on the centre-side penumbra (inclination with respect to the line-of-sight $\theta_{\mathrm{LOS}}<90^{\circ}$ ) of sunspots; on the limb-side penumbra $\left(\theta_{\mathrm{LOS}}>90^{\circ}\right)$ they found a preference for the flows to be situated in dark filaments. An explanation for this behaviour might be given by "differential opacity effects" (cf. Schlichenmaier et al. 2004; Westendorp Plaza et al. 2001), i.e. different line formation heights and, since the flows are not horizontal, by changing line-of-sight flow components from the limb- to the center-side penumbrae of sunspots. In our analysed data the penumbral filaments are oriented almost perpendicularly to the solar radius vector (cf. Fig. 2). Therefore, this differential opacity effect should be weak. However, in the penumbral model presented by Schlichenmaier et al. (1998) the flux tubes that carry the Evershed flow are hotter than the surroundings in the inner penumbra but this temperature excess diminishes in the outer penumbra. The "outer" penumbra in our data is represented by the close proximity to a dark, i.e. cool pore. Thus, it may be assumed that the flow channels are even cooler than the surrounding atmosphere. A dislocation of the aspect of the flow channels from bright filaments close to pore 1 to dark ones close to pore 2 might be taken into consideration.

The variation of the Stokes $V$ profiles across the FOV confirms the model assumption of horizontal flux tubes embedded in an inclined background field (Schlichenmaier et al. 1998; Müller et al. 2002): Close to pore 1 the blue lobe of the $V$ profiles produced by the background field is cancelled by the red lobe of the blue shifted $V$ profile of the flux tube component. Close to pore 2 the red lobe of the background field component 
is canceled by a red shifted flux tube component. In the transition of these two regions even more field components might be assumed in the resolution element; however, since our data are not corrected for instrumental polarisation effects only a tendency toward a qualitative agreement with former results (e.g. Schlichenmaier \& Collados 2002) can be asserted.

Acknowledgements. The Vacuum Tower Telescope is operated by the Kiepenheuer-Institut für Sonnenphysik in Freiburg (Germany) at the Spanish Observatorio del Teide of the Instituto de Astrofísica de Canarias in Tenerife. We are grateful to the anonymous referee for valuable comments. Financial support by the European Union (OPTICON Trans-National Access Programme) is gratefully acknowledged. This research is supported by a grant from the Austrian Fonds zur Förderung der wissenschaftlichen Forschung (ErwinSchrödinger-Rückkehrprogramm R11). K.G.P. thanks the Deutsche Forschungsgemeinschaft for support through grant KN 152/29-1 and J.J. and M.S. gratefully acknowledge support by the Grant Agency of the Academy of Sciences of the Czech Republic (grant IAA-3003404) and by the European Solar Magnetism Network (HRPN-CT-200200313).

\section{References}

Abdussamatov, H. I. 1976, Sol. Phys., 48, 117

Beckers, J. M., \& Schröter, E. H. 1969, Sol. Phys., 10, 384

Bello González, N., Okunev, O. V., Domínguez Cerdeña, I., Kneer, F., \& Puschmann, K. G. 2005, A\&A, 434, 317

Bellot Rubio, L. R., Balthasar, H., \& Collados, M. 2004, A\&A, 427, 319

Bendlin, C., Volkmer, R., \& Kneer, F. 1992, A\&A, 257, 817

Borrero, J. M., Solanki, S. K., Bellot Rubio, L. R., Lagg, A., \& Mathew, A. K. 2004, A\&A, 422, 1093

Choudhuri, A. R. 1986, ApJ, 302, 809

de Boer, C. R. 1993, Ph.D. Thesis, Universität Göttingen

Degenhardt, D., \& Wiehr, E. 1991, A\&A, 252, 821

Degenhardt, D., \& Lites, B. W. 1993, ApJ, 404, 383

Grigorjev, V. M., \& Katz, J. M. 1972, Sol. Phys., 122, 119

Grossmann-Doerth, U., Schmidt, W., \& Schröter, E. H. 1986, A\&A, 156,347

Hirzberger, J. 2003, A\&A, 405, 331

Hirzberger, J., \& Kneer, F. 2001, A\&A, 378, 1078

Hofmann, J., Deubner, F.-L., Fleck, B., \& Schmidt, W. 1994, A\&A, 284, 269

Johannesson, A. 1993, A\&A, 273, 633

Knölker, M., \& Schüssler, M. 1988, A\&A, 202, 275

Koschinsky, M., Kneer, F., \& Hirzberger, J. 2001, A\&A, 365, 588

Krieg, J., Wunnenberg, M., Kneer, F., Koschinsky, M., \& Ritter, C. 1999, A\&A 343, 983

Lites, B. W., Scharmer, G. B., \& Skumanich, A. 1990, ApJ, 355, 329

Lites, B. W., Socas-Navarro, H., Skumanich, A., \& Shimizu, T. 2002, ApJ, 575, 1131
Martínez Pillet, V. 2000, A\&A, 361, 734

Meyer, F., \& Schmidt, H. U. 1968, Z. Angew. Math. Mech., 4, T218

Montesinos, B., \& Thomas, J. H. 1993, ApJ, 402, 314

Montesinos, B., \& Thomas, J. H. 1997, Nature, 390, 485

Muller, R. 1973, Sol. Phys., 29, 55

Müller, D. A. N., Schlichenmaier, R., Steiner, O., \& Stix, M. 2002, A\&A, 393, 305

Parker, E. N. 1979, ApJ, 230, 905

Rimmele, T. R. 1995a, A\&A, 298, 260

Rimmele, T. 1995b, ApJ, 445, 511

Rouppe van der Voort, L. H. M., Löfdahl, M. G., Kiselman, D., \& Scharmer, G. B. 2004, A\&A, 414, 717

Sánchez Almeida, J. 1998, ApJ, 497, 967

Sánchez Almeida, J. \& Lites, B. W. 1992, ApJ, 398, 359

Sánchez Almeida, J., \& Martínez Pillet, V. 1994, ApJ, 424, 1014

Sánchez Almeida, J., \& Bonet, J. A. 1998, ApJ, 505, 1010

Scharmer, G. B., Gudiksen, B. V., Kiselman, D., Löfdahl, M. G., \& Rouppe van der Voort, L. H. M. 2002, Nature, 420, 151

Schlichenmaier, R., Jahn, K., \& Schmidt, H. U. 1998, A\&A, 337, 897

Schlichenmaier, R., \& Collados, M. 2002, A\&A, 381, 668

Schlichenmaier, R., Bellot Rubio, L. R., \& Tritschler, A. 2004, A\&A, 415,731

Schmidt, W., Hofmann, A., Balthasar, H., Tarbell, T. D., \& Franck, Z. A. 1992, A\&A, 264, L27

Schröter, E. H. 1965, Z. Astrophys., 62, 256

Semel, M. 1967, Annales d'Astophysique, 30, 513

Shine, R. A., Title, A. M., Tarbell, T. D., Smith, K., \& Frank, Z. A. 1994, ApJ, 430, 413

Sobotka, M., Brandt, P. N., \& Simon, G. W. 1997, A\&A, 328, 682

Sobotka, M., Brandt, P. N., \& Simon, G. W. 1999, A\&A, 348, 621

Sobotka, M., \& Sütterlin, P. 2001, A\&A, 380, 714

Solanki, S. K. 2003, A\&AR, 11, 153

Solanki, S. K., \& Montavon, C. A. P. 1993, A\&A, 275, 283

Stangl, S., \& Hirzberger, J. 2005, A\&A, 432, 319

Stellmacher, G., \& Wiehr, E. 1971, Sol. Phys., 17, 21

Sütterlin, P. 2001, A\&A, 374, L21

Thomas, J. H. 1988, ApJ, 333, 407

Title, A. M., Tarbell, T. D., Topka, K. P., et al. 1989, ApJ, 336, 475

Title A. M., Frank, Z. A., Shine, R. A., et al. 1993, in Sunspots: Theory and Observatons, ed. J. H. Thomas, \& N. O. Weiss (Dordrecht: Kluwer), 195

Tönjes, K., \& Wöhl, H. 1982, Sol. Phys., 75, 63

Volkmer, R. 1995, Ph.D. Thesis, Universität Göttingen

von der Lühe, O. 1984, J. Opt. Soc. Am. A1, 510

Weigelt, G. P. 1977, Optics Comm., 21, 55

Westendorp Plaza, C., del Toro Iniesta, J.C., Ruiz Cobo, B., et al 2001, ApJ, 547, 1130

Wiehr, E. 2000, Sol. Phys., 197, 227

Wiehr, E., \& Stellmacher, G. 1989, A\&A, 225, 528

Yi, Z., \& Molowny Horas, R. L. 1992, in Proc. LEST Mini-Workshop, Software for Solar Image Processing, ed. Z. Yi, T. Darvann, \& R. Molowny Horas, Oslo, Inst. f. Theoretical Astrophys., 69 